\title{
A method for the definition of a self-awareness behavior dimension with clinical subjects: A latent trait analysis
}

\author{
Stefania Mannarini \\ University of Padua, Padua, Italy
}

\begin{abstract}
The main scope of the present study was to devise a method in order to define a dimension characteristic of self-awareness behaviors with clinical subjects. To do so, I adopted a latent trait methodological approach. I studied the way patients expressed their treatment requests through their behaviors, both during their admission to a medical center in Northern Italy and after a period of treatment that involved an integrated (psychoanalytical and pharmacological) approach. The subjects were 48 females suffering from affective disorders, often combined with personality disorders. Five self-awareness indicators were identified, based both on interviews conducted with the patients and on the literature on the subject. The data gathered were analyzed by means of the many-facet Rasch model (Linacre, 1989). The results confirmed the existence of a self-awareness dimension characterized by the five indicators. Moreover, there was evidence that an improvement in self-awareness occurred during the pretreatment to posttreatment time period for both the affective disorders with personality problems patients and the affective disorders without personality problems patients. The estimation of bias/interactions showed the existence of specific behavioral differences between the two groups of patients. This study demonstrates the appropriateness of the methodological tool adopted, opening new expectations with regard to the integration of two approaches - psychoanalytical and pharmacological ones - in the treatment of psychiatric subjects.
\end{abstract}

The term awareness is defined as "a condition of being conscious of something." It follows that the expression self-awareness indicates the condition of being conscious of oneself (e.g., Silvestri et al., 2008). Two concepts, then, are involved in the definition of awareness and self, and both are long since the issues of scientific discussions, mainly philosophical, but also psychological and clini$\mathrm{cal}$. The debate concerning self-awareness from a clinical point of view is often carried out by psychiatrists, who define such a concept with the term insight (e.g., Markova \& Berrios, 1995). Lack of insight is generally considered to be a symptom of psychosis, and from this perspective, many studies are developed with the intent of defining specific devices for the assessment of different levels of insight in schizophrenic patients (e.g., Birchwood et al., 1994; Markova \& Berrios, 1992, 1995).

In the present study, the self-awareness concept is approached from a psychoanalytic point of view with psychiatric patients (Silvestri et al., 2008). As is well known, the psychoanalytic approach is concerned with the analysis of the subjectivity of the patient; the psychoanalyst does not primarily address the symptomatic manifestations of the patient (S. Freud, 1914/1975), but his/her aim consists in the development of the patient's latent abilities, such as sense of responsibility, autonomy, self-consciousness, and capacity for communicating with others (Lopez, 1983; Lopez \& Zorzi Meneguzzo, 1989). Thus, the development of self-awareness behaviors becomes the main objective of a psychoanalytically oriented therapy. A discussion on the importance of self-awareness in psychoanalysis was carried out in the past and still is, in the present day (e.g., Blum, 1979; A. Freud, 1979; Hatcher, 1973; Kennedy, 1979; Kris, 1975; Mangini, 2001; Neubauer, 1979; Rangell, 1981; Sacerdoti \& Spacal, 1985; Sandler, 1979), but so far no study seems to exist that develops specific psychometrically tested methods that allow the operationalization of the self-awareness concept in view of a convincing representation of it.

The necessity of devising a mathematically and statistically valid method for the definition of a self-awareness behavior dimension is the main scope of the present research. I begin with a definition of awareness of self as "a perspective space where the individual organizes his/her own self-representation" (Silvestri et al., 2008, p. 378). The self-awareness organizing process, then, is the capacity of feeling, understanding, and communicating the link between what happens inside and outside oneself (Mangini, 2001; Sacerdoti, 1986); that is to say, the individual inside and outside worlds are related, and moreover, each individual is part of a network of other individuals.

On the basis of the above definition of self-awareness, the specific objectives of this study are as follows: (1) the definition of a set of self-awareness behavior indices; 
(2) the definition of a self-awareness behavior dimension specified by the indices, named psychiatric patients'selfawareness (PPSA); (3) the analysis of possible interactions between specific self-awareness behavior indices and types of diagnoses; (4) the analysis of self-awareness behavior indices as change might occur in a period of time; (5) the definition of the probability of occurrence of each index on the PPSA dimension; and (6) the definition of the probability that a patient with a specific selfawareness level is located on the PPSA dimension.

After the self-awareness behavior indices are defined, the application of the many-facet Rasch model (MFRM; Linacre, 1989) in the following paragraphs will allow the pursuit of the above-mentioned objectives from a latent trait perspective. Considering the psychoanalytic approach of this study, a latent trait analysis by means of the MFRM seems particularly appropriate for the following reasons: (1) The MFRM analysis of the subjects $X$ indices matrix makes it possible to gather evidence about whether a latent dimension where the self-awareness indices are located exists; (2) the MFRM analysis makes it possible to investigate, at a latent level, whether self-awareness behaviors might be affected by variables such as diagnosis and time; and (3) the MFRM allows the computation of probability values for each subject and for each index that combine subject and index on the self-awareness dimension, given a specific diagnosis and a specific time.

\section{METHOD}

\section{Procedure and Subjects}

In order to define a set of specific indices typical of self-awareness behaviors according to the definition given above, in this study a team of experts (psychiatrists, psychoanalysts, and psychologists) evaluated 48 patients on two successive occasions: pretreatment, when the medical team visited the patient upon her arrival at the medical center; and posttreatment, when the patient was revisited after a period of time that varied from 20 to 88 days. With each patient, an interview was conducted on both occasions and the collected material was revised and discussed by the medical team. On the basis of an accurate and deep revision of the interviews and of the literature as well (e.g., Birchwood et al., 1994; Markova \& Berrios, 1995; Roncone et al., 2003), five indices were devised, as follows.

1. Request. The patient decides autonomously to ask for help. The lower the awareness of self is in the patient, the less probable it is that the patient will behave autonomously in asking for medical care.

2. Autonomy. The patient is aware of his/her own health status. The patient behaves autonomously when replying to the others' decision to ask for medical care, either refusing or accepting it.

3. Content. The content consists of the reasons why the request for help is advanced. The patient behaves as expected in relation to the motivations that the others, such as relatives, have shown when asking for medical care.

4. Relations. The patient is able to communicate with the others involved. The patient behaves appropriately in relation to the others, showing interest in what the others do and in their attitudes.

5. Context. The patient is aware of the context in which he/she is acting. The patient behaves appropriately in relation to the situation and the environment that he/she is dealing with at the medical center.

After the five indices were defined, each patient was evaluated on a rating scale, from 0 to 4 , in relation to each index, on two successive occasions, as mentioned above. A score of 0 meant that an index was not present, a score of 1 meant it was very slightly present, 2 meant it was slightly present, 3 meant it was quite present, and 4 meant it was totally present. A patients $\times$ indices matrix was then obtained, at each time, which was specified by a random variable representing self-awareness. This variable could have values ranging from 0 to 4 .

The subjects were 48 Italian adult females, ${ }^{1}$ mean age 50.29 years. Their marital status was married (54\%), single (23\%), divorced $(17 \%)$, and widowed $(6 \%)$. Their school levels were low $(65 \%)$, medium (30\%), and high (5\%). All of the subjects were affected by affective disorders and were classified according to two diagnoses: with and without personality problems (affective disorders with personality problems, ADP, and affective disorders without personality problems, ADWP). As explained above, each patient was visited by a team of experts on two successive occasions: pretreatment, upon the subject's arrival at the medical center, and posttreatment, after a period of time. During this period, each patient was supposed to undertake a therapy based on the integration of two approaches, a psychoanalytic and a pharmacological one (Silvestri et al., 2008).

\section{The Model}

As explained in the literature (Linacre, 1989), in an MFRM approach, each observation is conceptualized to be the outcome of an interaction between elements, such as, for instance, the ability of a person, the difficulty of an item, and the severity of a rater. By means of an MFRM approach, these interacting elements or facets are modeled to operate independently and so that their measures can be combined additively on a latent variable. The MFRM is considered to be an extension of the Rasch simple logistic model (RSLM; Rasch, 1960/1980). The RSLM focuses on two elements - namely, on two facets of the latent trait being measured: the ability of a person and the difficulty of an item. However, more complex measurement situations exist in which other aspects interfere between an attribute characteristic of a person and an attribute characteristic of an item, such as specific social, educational, or clinical attributes that characterize the persons, or different occasions on which the items are administered to the persons, or different methods applied to administer the items, and so on. Within the unidimensionality of Rasch modeling, Linacre (1989) developed the MFRM, which takes into account these more complex situations.

The original RSLM - with two facets, where the probability of any answer is a function of the ability (Facet 1 ) of the person and the difficulty (Facet 2) of the item - takes the form of Equation 1 (Rasch, 1960/1980):

$$
P\left(X_{n i}=x\right)=\frac{e\left(\beta_{n}-\delta_{i}\right)}{1+e\left(\beta_{n}-\delta_{i}\right)},
$$

where $P\left(X_{n i}=x\right)$ is the probability of the response $x$ of person $n$ to item $i, \beta_{n}$ is the parameter that denotes the ability of person $n$, and $\delta_{i}$ is the parameter that denotes the difficulty of item $i$. Equation 1 can be formulated also in logit form by means of Equation 2 (Rasch, 1960/1980):

$$
\ln \left(\frac{P\left(X_{n i}=x\right)}{1-P\left(X_{n i}=x\right)}\right)=\beta_{n}-\delta_{i} .
$$

The MFRM, with which the probability of any answer is a function not only of two facets, but of more elements, takes the form of Equation 3 (Linacre, 1989). The elements taken into consideration in this study are combined: the patient's self-awareness (Facet 1), the self-awareness index (Facet 2), the two times (Facet 3), and the types of diagnosis (Facet 4). This results in

$$
P\left(X_{\text {nitdk }}\right)=\frac{e\left[\beta_{n}-\delta_{i}-\tau_{t}-\varpi_{d}-\varphi_{k}\right]}{1+e\left[\beta_{n}-\delta_{i}-\tau_{t}-\varpi_{d}-\varphi_{k}\right]},
$$

where $\beta_{n}$ is the person $n$ measure, $\delta_{i}$ is the index $i$ measure, $\tau_{t}$ is the time $t$ measure, $\varpi_{d}$ is the diagnosis $d$ measure, and $\varphi_{k}$ is the measure 
of the step up to category $k$ of the rating scale. Equation 3 in logit takes the form of Equation 4 (Linacre, 1989):

$$
\ln \left(\frac{P\left(X_{\text {nitdk }}=x\right)}{1-P\left(X_{\text {nitdk }}=x\right)}\right)=\beta_{n}-\delta_{i}-\tau_{t}-\varpi_{d}-\varphi_{k} .
$$

All the facet measures are estimated simultaneously, so they can be considered separately. The measure estimation procedure adopted in the Facets program (Linacre, 2005) is designed to handle the more complex applications of unidimensional Rasch measurement and perform the measure estimation by means of the joint maximum likelihood estimation procedure. For all facet estimates, except for subjects, negative measures indicate higher levels of self-awareness, whereas positive measures indicate the opposite. This is also true for bias/interaction measures. With regard to the control fit evaluation of the measures, as is well known, the fit statistics must determine how well any set of empirical data meets the requirements of the model. In the output of the Facets program, two fit statistics are reported for each measure of a facet, the mean square outfit statistic and the mean square infit statistic. The outfit and infit statistics have a scale form with an expected value of +1 and a range from 0 to positive infinity. An outfit or infit statistic in the range .70-1.30 indicates a satisfactory fit of the empirical data to the model. The explanation is that an infit or outfit mean square value of $1+x$ indicates $100 x \%$ more variation between the observed and the model-predicted response patterns than would be expected if the data and the model were perfectly compatible. Thus, an infit statistic, for instance, of 1.30 indicates $30 \%$ more variation in the observed data than the model predicted. The value .70 indicates $30 \%$ less variation in the observed data than the model predicted (Bond \& Fox, 2001).

A chi-square statistic is also calculated (the fixed chi-square) in relation to each facet, in order to verify whether its elements (e.g., the five indices of Facet 2, in this study) are equal or they are significantly different - that is, whether they measure different aspects of a facet or not.

After estimating the measures, the Facets program can also check for biases for a given model. A bias can be due to any kind of interaction, such as differential item functioning, differential person functioning, or differential functioning of any other facet, and is estimated from the residuals left over after estimating the measures in the main analysis (Linacre, 2005). Detecting a bias serves many purposes in the research, and is of particular importance when, in the frame of a Rasch analysis, possible differences between specific levels of facets might influence the interpretation of the results. For example, if an interaction/bias associated to a specific self-awareness index and the diagnosis of persons were to be detected, it would mean that a residual remains after estimating the index measure from which two new measures, one for Diagnosis 1 and one for Diagnosis 2, can be estimated. Such measures can be compared by means of the $t$ statistic. If the $t$ statistic is significant ( $p=.05$ ), then the diagnosis of persons affects the index or, similarly, the index has a differential functioning in relation to diagnosis. In this study, after estimating the measures for all the facets, the interaction/biases for time and diagnosis will be analyzed according to the main objectives formulated above.

\section{RESULTS}

\section{Facet Measures}

The data show a mean of .44 and a standard error of .40 , for the person measures $\left(\beta_{n}\right)$. The infit and outfit statistics are satisfactory, being, respectively, 1.01 and 1.00. The range for person measures is -2.23 to 2.16 and the range for standard errors is .38-.50. In Table 1, the index measures $\left(\delta_{i}\right)$, the standard errors, and the infit and out-
Table 1

Index Measures, Standard Errors, and Infit and Outfit Statistics

\begin{tabular}{|c|c|c|c|c|}
\hline Index & $\begin{array}{l}\text { Measure } \\
\text { Statistic }\end{array}$ & $S E$ & Infit & Outfit \\
\hline 1. Request & -.88 & .13 & 1.70 & 1.69 \\
\hline 2. Autonomy & -.61 & .13 & 1.09 & 1.09 \\
\hline 3. Content & .32 & .13 & 0.70 & 0.70 \\
\hline 5. Context & .34 & .13 & 0.70 & 0.72 \\
\hline 4. Relations & .83 & .13 & 0.78 & 0.80 \\
\hline
\end{tabular}

fit statistics are presented. Such results were obtained for pre- and posttreatments and for diagnosis groups.

As shown, the fit statistics are good, with the exception of Index 1 (request), where both statistics slightly overcome the limits (Bond \& Fox, 2001). In general, the results indicate that the five indices are satisfactorily located on the latent variable named PPSA, as expected. The fixed chi-square is 123.3, with 4 degrees of freedom and probability .00 . Such a result indicates that the five indices measure different aspects of the PPSA dimension. Analyzing the index measures, it appears that Index 1 (request) is most frequently attributed to the patients $(-.88)$ and that Index 4 (relations) is the least present (.83).

Supplementary analyses concerning request, suggested by the misfit of this index, show that it does not fit the model — both when the analyses are carried out separately in the pre- and posttreatment situations and for the two groups - in that the infit and outfit statistics are in the range 1.68-1.77. The misfit of Index 1 does not seem to confirm the expectation that the lower the awareness of self is in the patient, the less probable it is that the patient will behave autonomously in asking for medical care. In other words, it seems that levels of self-awareness and autonomy in the request for help do not behave coherently. Hypotheses were formulated in order to interpret such a result. In particular, the experts tended to retain the hypothesis of a relation between selfawareness and a request for care and to think that the misfit of Index 1 was mainly due to both some inadequacy of the interview with regard to such an index and to an unsatisfactory interpretation of the material collected during the interview. More questions in the interviews with the patients and a more accurate examination of the responses as far as request is concerned are planned in view of future analyses and of a definitive definition of the self-awareness dimension.

In Table 2, the measures, the standard errors, and the infit and outfit statistics for pre- and posttreatment $\left(\tau_{t}\right)$ and diagnosis groups $\left(\varpi_{d}\right)$ are presented. As shown, the fit statistics are all satisfactory, both for pre- and posttreatment occasions and for diagnosis groups, indicating that assessments of self-awareness in the pretreatment and posttreatment situations result in two measures that are coherently located on the PPSA dimension. This is true for the diagnoses as well; that is, the two awareness measures related to the diagnosis groups are also properly located on the PPSA dimension. The fixed chi-square is 17.6 , with 1 degree of freedom and probability .00 for pre- 
Table 2

Two Facets: Time and Diagnosis, Measures, Standard Errors, and Infit and Outfit Statistics

\begin{tabular}{llcccc}
\hline Facet & & Measure & $S E$ & Infit & Outfit \\
\hline Time & Pre & .24 & .08 & 0.95 & 0.96 \\
& Post & -.24 & .08 & 1.02 & 1.03 \\
\multirow{2}{*}{ Diagnosis } & ADP & -.03 & .09 & 0.99 & 1.08 \\
& ADWP & .03 & .07 & 0.99 & 0.98 \\
\hline
\end{tabular}

Note-Pre, upon arrival at the medical center; Post, after a period of treatment; ADP, affective disorders with personality problems; ADWP, affective disorders without personality problems.

$(.24)$ and post- $(-.24)$ treatment measures, showing that a significant change occurs in the period of time considered. Self-awareness improves after a period of treatment, considering all indices and both diagnoses. In order to better analyze the pre- and posttreatment difference, a plot of pre- versus posttreatment patient locations is presented in Figure 1, where the index measures are anchored.

The plot presented in Figure 1 confirms the result that significant change occurred in the period of time from pretreatment through posttreatment. Pretreatment, 56\% of patients are located above 0 on the logit scale, $6 \%$ are at 0 , and $38 \%$ are below 0 , whereas in the posttreatment situation, $73 \%$ of patients are above $0,2 \%$ are at 0 , and $25 \%$ are below 0 .

With regard to diagnoses, the results show that the ADP patients' measure is - .03 and the ADWP patients' measure is .03; the fixed chi-square is .20 with 1 degree of freedom and probability .63. This result shows that self-awareness does not differ in relation to the two diagnoses, considering all indices and the period of time pre- and posttreatment.

In Figure 2, a variable map is presented as a summary of the results. Figure 2 makes it possible to appreciate the ranges of the four facets and to make an immediate comparison of the different facet ranges. In general, the facet distributions match quite satisfactorily, except for the person distribution, which is rather top-heavy in comparison with the index distribution; in fact, $67 \%$ of the patients are above 0 on the logit scale, $10 \%$ are at 0 , and $23 \%$ are below 0 .

\section{Bias/Interaction Analysis}

In Table 3, the interactions between self-awareness and the two diagnoses, both in the pre- and posttreatment situations, are considered. The findings show that selfawareness behaviors differ in relation to Index 2 (autonomy) on both occasions - that is, when the patient enters the medical center $(p=.0051)$ and after a period of treatment $(p=.0476)$.

The direction of the differences indicates that upon arrival, self-awareness is worse for ADWP patients $(0.27)$ than for ADP patients $(-0.83)$ but that after a period of treatment, self-awareness is better for ADWP patients $(-1.31)$ than for ADP $(-0.52)$ patients. Another bias/interaction is concerned with Index 5 (context) in the posttreatment situation $(p=.0201)$, in which the ADWP patients are shown to behave more properly $(-0.41)$ than the $\operatorname{ADP}(0.48)$ patients as far as the situation and the environment they are dealing with at the medical center are concerned.
Further considering the interactions between selfawareness and the two occasions, pre- and posttreatment, for the two diagnoses separately, the results are significant again in relation to Index 2 (autonomy), both for ADP $(p=.017)$ and $\operatorname{ADWP}(p=.016)$ patients. The direction of the differences indicates that the ADP patients seem to be more aware $(-1.10)$ of their health status upon their arrival at the medical center than after a period of treatment $(-0.31)$, whereas for the ADWP patients, the opposite situation is observed; that is, after a period of treatment, their self-awareness has improved -0.05 in the pre- and -1.04 in the posttreatment situation, respectively.

Considering the data in Table 3 in particular, as far as Index 2 (autonomy) is concerned, hypotheses are advanced in order to interpret the behavior of the ADP group. When entering the medical center, patients with affective disorders and personality problems are more aware of their health status than are the ADWP patients; nevertheless, their awareness seems either to decrease or not to improve after a period of treatment. According to the experts' opinions, the ADP patients appear to be more conscious and autonomous than the ADWP patients when replying to the others' decisions about asking for medical care in the pretreatment situation, whereas their real health condition, as far as self-awareness is concerned, might be quite critical. It is by means of an appropriate treatment that the real nature of the ADP patients' awareness can be put into evidence from a clinical point of view. In Table 3, the results obtained in the posttreatment situation demonstrate this.

\section{Probabilities}

For each patient, by applying the formula of Equation 3, an MFRM probability value is calculated for each score of the rating scale from 0 to 4 , both at Time 1 and at Time 2, taking into consideration the two diagnoses separately. In order to apply Equation 3, the measures of the steps up to the categories $k$ of the rating scale $\left(\varphi_{k}\right)$ are also taken into consideration, which are -2.37 (up to Category 1), -0.53 (up to Category 2), 1.03 (up to Category 3), and 1.86 (up to Category 4). In Tables 4 and 5, two examples of such probabilities are presented. Table 4 shows the five index measures, the patient's average rating on the five indices (range $0-4$ ), and the corresponding patient measure at Time 1, separately for the two diagnoses, for the ADP patients (Table 4A) and the ADWP patients (Table 4B), respectively. For illustrative purposes, each cell of the tables contains the probability of presenting an index that is rated 3 by the experts, given the index measure on the PPSA self-awareness behavior dimension, the patient measure over all the indices, and the diagnosis (ADP and ADWP, respectively) measure, in the pretreatment situation. Tables 5A and 5B illustrate the same situations as do Tables $4 \mathrm{~A}$ and $4 \mathrm{~B}$, but at Time 2 - that is, after a period of treatment.

In order to interpret the data in Tables 4 and 5, it is useful to point out that if at Time 1 (.24 measure) a patient with an overall self-awareness mean rating of 1.7 (patient measure -.33$)$, with diagnosis ADP $(-.03)$, is rated on 


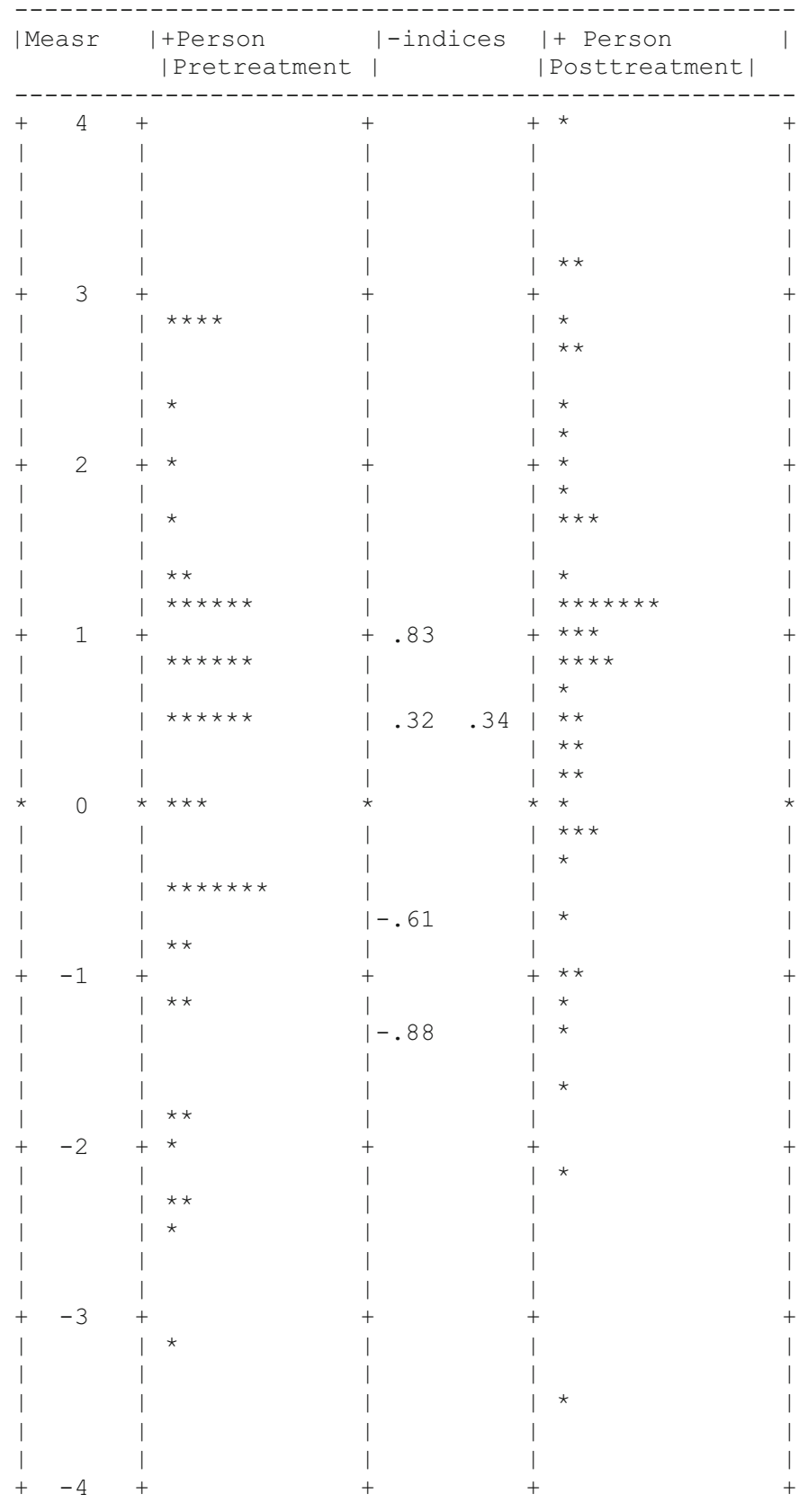

Figure 1. Plot of pre- versus posttreatment persons' locations with anchored index measures.

Index $1(-.88)$ in Category $3(1.03$, quite present), then the probability value is

$$
\frac{e[-.33-.24-(-.88)-(-.03)-1.03]}{1+e[-.33-.24-(-.88)-(-.03)-1.03]}=0.32
$$

(see the bold cells in Table 4A). This result shows that the rating of 3 for Index 1 (the patient decides autonomously to ask for help) has to be considered quite usual, because its relative probability of occurrence on the PPSA vari- able is rather high $(p=.32)$. But the same cannot be said when a patient is rated 3 on Index 4 (the patient is able to communicate with the others) because this has a low probability $(p=.08)$, with a mean score of 1.7 . A look at the probabilities values in the two tables shows that at Time 1 (Table 4) the probabilities are lower than at Time 2 (Table 5), and within each table, the probabilities for the ADP patients are slightly higher than the probabilities for the ADWP patients. This result confirms that there is an improvement in the subjects' self-awareness behavior in 


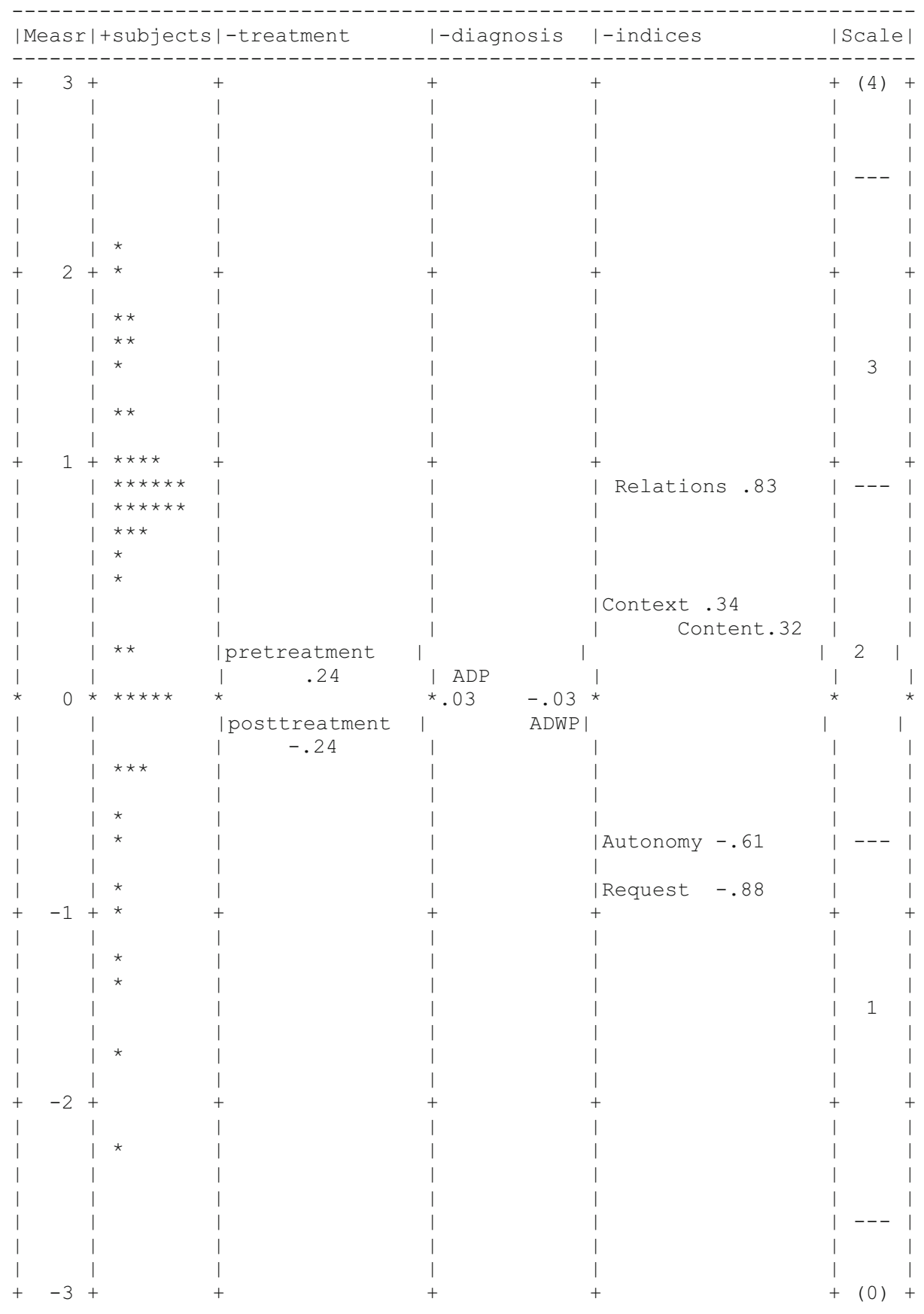

Figure 2. Facet map. Logit scale measures, subject measures, pre- and posttreatment measures, diagnosis group measures, index measures, and rating scale.

the period of time considered; that is, at Time 2, the probabilities associated to the self-awareness indices are in general higher.

\section{REMARKS AND CONCLUSION}

The main purpose of the present study was to address the problem of defining a mathematically and statistically valid method for evidencing and measuring a self-awareness behavior dimension with clinical subjects. In the literature, specific problems regarding measurement procedures in clinical psychology are often debated, since the need to define specific clinical behavior indices, the interest in analyzing possible interactions between such indices and types of diagnoses, and the necessity of analyzing whether particular behavior indices might change during a period 
Table 3

Bias/Interactions: Indices by Pre-Post Treatment and Diagnoses; Measures and Fit Probabilities

\begin{tabular}{|c|c|c|c|c|c|c|c|c|c|c|c|c|}
\hline & \multicolumn{6}{|c|}{ Time } & \multicolumn{6}{|c|}{ Diagnosis } \\
\hline & \multicolumn{3}{|c|}{ Pre } & \multicolumn{3}{|c|}{ Post } & \multicolumn{3}{|c|}{ ADP } & \multicolumn{3}{|c|}{ ADWP } \\
\hline & ADP & ADWP & $p$ & ADP & ADWP & $p$ & Pre & Post & $p$ & Pre & Post & $p$ \\
\hline $\begin{array}{l}\text { Index } 2 \\
\text { Index } 5\end{array}$ & -0.83 & 0.27 & .0051 & $\begin{array}{l}-0.52 \\
-0.41\end{array}$ & $\begin{array}{r}-1.31 \\
0.48\end{array}$ & $\begin{array}{l}.0476 \\
.0201\end{array}$ & -1.10 & -0.31 & .017 & 0.05 & -1.04 & .016 \\
\hline
\end{tabular}

Note-Index 2, autonomy; Index 5, context. Pre, upon arrival at the medical center; Post, after a period of treatment. ADP, affective disorders with personality problems; ADWP, affective disorders without personality problems.

of time are only some of the issues for which satisfactory methodological solutions have not yet been found.

The method chosen and applied in this investigation was the MFRM (Linacre, 1989). Such a method-in line with the specific objectives of this study, as outlined above, and within a latent trait perspective - has proved to be very efficient in allowing interpretable results such as the definition of a set of self-awareness behavior indices; the definition of a self-awareness behavior dimension specified by the indices; the definition of interactions between specific self-awareness behavior indices and types of diagnoses; the analysis of self-awareness behavior indices in a period of time; the definition of the probability of occurrence of each index on the dimension; and the definition of the probability that a patient with a specific self-awareness level is located on the dimension.
The subjects were 48 adult females, mean age 50.29 years. All of the subjects presented affective disorders and were classified according to two diagnoses: with and without personality problems (ADP and ADWP, respectively). In order to define a set of specific indices typical of self-awareness behaviors, a team of experts (psychiatrists, psychoanalysts, and psychologists) evaluated the 48 subjects on two successive occasions: at Time 1, when the medical team visited the patient upon her arrival at the medical center, and at Time 2 , when the patient was revisited after a period of time that varied from 20 to 88 days. With each patient, an interview was conducted on both occasions and the collected material was revised and discussed by the medical team. Five indices were devised: the patient's request for care, the autonomy of the patient's behavior, the content of the request, the patient's relations

Table 4

Probability Values of Self-Awareness Indices Calculated for Score 3 (Step Measure 1.03) on the Rating Scale 0 to 4, Pretreatment (Measure $=.24$ )

\begin{tabular}{|c|c|c|c|c|c|c|c|c|c|c|c|}
\hline \multirow{5}{*}{$\begin{array}{c}\text { Patient's } \\
\text { Average } \\
\text { Rating } \\
\text { (on 5 } \\
\text { Indices; } \\
\text { Range 0-4) } \\
\end{array}$} & \multicolumn{6}{|c|}{$\begin{array}{c}\text { A. } \\
\text { ADP Measure }=-.03\end{array}$} & \multicolumn{5}{|c|}{$\begin{array}{c}\text { B. } \\
\text { ADWP Measure }=.03\end{array}$} \\
\hline & & \multicolumn{5}{|c|}{ Index Measure } & \multicolumn{5}{|c|}{ Index Measure } \\
\hline & & Request & Autonomy & Content & Context & Relations & Request & Autonomy & Content & Context & Relations \\
\hline & Patient & 1 & 2 & 3 & 5 & 4 & 1 & 2 & 3 & 5 & 4 \\
\hline & Measure & $(-.88)$ & $(-.61)$ & $(.32)$ & $(.34)$ & $(.83)$ & $(-.88)$ & $(-.61)$ & $(.32)$ & $(.34)$ & $(.83)$ \\
\hline 3.3 & 2.16 & .85 & .81 & .63 & .63 & .51 & .86 & .82 & .65 & .64 & .52 \\
\hline 3.2 & 1.97 & .83 & .78 & .59 & .58 & .46 & .83 & .79 & .60 & .60 & .48 \\
\hline 3.1 & 1.79 & .80 & .75 & .54 & .54 & .42 & .81 & .76 & .56 & .55 & .43 \\
\hline 3.0 & 1.63 & .77 & .72 & .50 & .50 & .38 & .78 & .73 & .52 & .51 & .39 \\
\hline 2.9 & 1.47 & .74 & .69 & .46 & .46 & .34 & .75 & .70 & .48 & .47 & .35 \\
\hline 2.8 & 1.31 & .71 & .65 & .42 & .41 & .31 & .72 & .66 & .44 & .43 & .32 \\
\hline 2.7 & 1.16 & .68 & .62 & .39 & .39 & .27 & .69 & .63 & .40 & .40 & .29 \\
\hline 2.6 & 1.01 & .64 & .58 & .35 & .35 & .25 & .65 & .59 & .37 & .36 & .26 \\
\hline 2.5 & 0.87 & .61 & .54 & .32 & .32 & .22 & .62 & .56 & .33 & .33 & .23 \\
\hline 2.4 & 0.72 & .58 & .51 & .29 & .28 & .20 & .59 & .52 & .30 & .30 & .21 \\
\hline 2.3 & 0.58 & .54 & .47 & .26 & .26 & .18 & .55 & .49 & .27 & .27 & .18 \\
\hline 2.2 & 0.43 & .50 & .44 & .23 & .23 & .15 & .52 & .45 & .24 & .24 & .16 \\
\hline 2.0 & 0.13 & .43 & .36 & .18 & .18 & .12 & .44 & .38 & .19 & .17 & .13 \\
\hline 1.9 & -0.02 & .39 & .33 & .16 & .16 & .10 & .41 & .34 & .17 & .17 & .11 \\
\hline 1.8 & -0.17 & .36 & .30 & .14 & .14 & .09 & .37 & .31 & .15 & .15 & .10 \\
\hline 1.7 & -0.33 & .32 & .27 & .12 & .12 & .08 & .33 & .28 & .13 & .13 & .08 \\
\hline 1.6 & -0.49 & .29 & .23 & .11 & .11 & .07 & .30 & .25 & .11 & .11 & .08 \\
\hline 1.5 & -0.65 & .26 & .21 & .09 & .09 & .06 & .27 & .22 & .10 & .10 & .06 \\
\hline 1.4 & -0.82 & .22 & .18 & .08 & .08 & .05 & .24 & .19 & .08 & .08 & .05 \\
\hline 1.3 & -1.00 & .19 & .16 & .07 & .07 & .04 & .20 & .16 & .07 & .07 & .04 \\
\hline 1.2 & -1.18 & .17 & .13 & .06 & .06 & .04 & .18 & .14 & .06 & .06 & .04 \\
\hline 1.1 & -1.37 & .14 & .11 & .05 & .05 & .03 & .15 & .12 & .05 & .05 & .03 \\
\hline 0.9 & -1.77 & .10 & .08 & .03 & .03 & .02 & .11 & .08 & .03 & .03 & .02 \\
\hline 0.7 & -2.23 & .07 & .05 & .02 & .02 & .01 & .07 & .05 & .02 & .02 & .01 \\
\hline
\end{tabular}

Note-Index measure values are in parentheses. Bold entries: At Time 1, for an ADP patient with an overall self-awareness average rating of 1.7 and a patient measure of -.33 , the probability of presenting Index request (in Category 3 ) is .32 . 
Table 5

Probability Values of Self-Awareness Indices Calculated for Score 3 (Step Measure $=1.03$ ) on the Rating Scale 0 to 4, Posttreatment (Measure $=-.24$ )

\begin{tabular}{|c|c|c|c|c|c|c|c|c|c|c|c|}
\hline \multirow{5}{*}{$\begin{array}{c}\text { Patient's } \\
\text { Average } \\
\text { Rating } \\
\text { (on 5 } \\
\text { Indices; } \\
\text { Range 0-4) } \\
\end{array}$} & \multicolumn{6}{|c|}{$\begin{array}{c}\text { A. } \\
\text { ADP Measure }=-.03\end{array}$} & \multicolumn{5}{|c|}{$\begin{array}{c}\text { B. } \\
\text { ADWP Measure }=.03\end{array}$} \\
\hline & & \multicolumn{5}{|c|}{ Index Measure } & \multicolumn{5}{|c|}{ Index Measure } \\
\hline & & Request & Autonomy & Content & Context & Relations & Request & Autonomy & Content & Context & Relations \\
\hline & Patient & 1 & 2 & 3 & 5 & 4 & 1 & 2 & 3 & 5 & 4 \\
\hline & Measure & $(-.88)$ & $(-.61)$ & $(.32)$ & $(.34)$ & $(.83)$ & $(-.88)$ & $(-.61)$ & $(.32)$ & $(.34)$ & $(.83)$ \\
\hline 3.3 & 2.16 & .90 & .88 & .74 & .73 & .62 & .91 & .88 & .74 & .74 & .64 \\
\hline 3.2 & 1.97 & .88 & .85 & .70 & .69 & .58 & .89 & .86 & .71 & .70 & .59 \\
\hline 3.1 & 1.79 & .86 & .83 & .66 & .65 & .54 & .87 & .84 & .67 & .67 & .55 \\
\hline 3.0 & 1.63 & .84 & .80 & .62 & .61 & .50 & .85 & .82 & .63 & .63 & .51 \\
\hline 2.9 & 1.47 & .82 & .78 & .59 & .58 & .45 & .83 & .79 & .60 & .59 & .47 \\
\hline 2.8 & 1.31 & .80 & .75 & .54 & .54 & .42 & .81 & .76 & .56 & .55 & .43 \\
\hline 2.7 & 1.16 & .77 & .72 & .51 & .50 & .38 & .78 & .73 & .52 & .52 & .39 \\
\hline 2.6 & 1.01 & .74 & .69 & .47 & .46 & .34 & .76 & .70 & .48 & .48 & .36 \\
\hline 2.5 & 0.87 & .72 & .66 & .43 & .43 & .31 & .73 & .67 & .45 & .44 & .33 \\
\hline 2.4 & 0.72 & .69 & .62 & .40 & .39 & .28 & .70 & .64 & .41 & .40 & .30 \\
\hline 2.3 & 0.58 & .65 & .59 & .36 & .36 & .26 & .67 & .61 & .38 & .37 & .27 \\
\hline 2.2 & 0.43 & .62 & .55 & .33 & .33 & .23 & .63 & .57 & .34 & .34 & .24 \\
\hline 2.0 & 0.13 & .55 & .48 & .27 & .26 & .18 & .56 & .50 & .28 & .27 & .19 \\
\hline 1.9 & -.02 & .51 & .44 & .24 & .24 & .16 & .53 & .46 & .25 & .25 & .17 \\
\hline 1.8 & -.17 & .47 & .41 & .21 & .21 & .14 & .49 & .42 & .22 & .22 & .15 \\
\hline 1.7 & -.33 & .43 & .37 & .19 & .18 & .12 & .45 & .38 & .19 & .19 & .13 \\
\hline 1.6 & -.49 & .39 & .33 & .16 & .16 & .11 & .41 & .35 & .17 & .17 & .11 \\
\hline 1.5 & -.65 & .36 & .30 & .14 & .14 & .09 & .37 & .31 & .15 & .15 & .10 \\
\hline 1.4 & -.82 & .32 & .26 & .12 & .12 & .08 & .33 & .27 & .13 & .13 & .08 \\
\hline 1.3 & -1.00 & .28 & .23 & .11 & .10 & .07 & .29 & .24 & .11 & .11 & .07 \\
\hline 1.2 & -1.18 & .25 & .20 & .09 & .09 & .06 & .26 & .21 & .09 & .09 & .06 \\
\hline 1.1 & -1.37 & .21 & .17 & .08 & .07 & .05 & .22 & .18 & .08 & .08 & .05 \\
\hline 0.9 & -1.77 & .15 & .12 & .05 & .05 & .03 & .16 & .13 & .06 & .05 & .03 \\
\hline 0.7 & -2.23 & .10 & .08 & .03 & .03 & .02 & .11 & .08 & .04 & .03 & .02 \\
\hline
\end{tabular}

Note-Index measure values are in parentheses.

with the others, and the patient's awareness of the context. Hypotheses were made that the patient's self-awareness could be evaluated in relation to the five indices and that such indices could be ordered on a dimension of selfawareness. Further hypotheses were concerned with the location of the patients on the dimension and with the interactions between types of diagnoses and self-awareness during the period of treatment considered.

The main results can be summarized as follows. Four out of the five indices contribute differently to define a dimension called psychiatric patients'self-awareness (PPSA). Index 1, request, does not fit the model satisfactorily, suggesting the need for more analyses. The indices' estimated measures can be placed on the dimension according to an increasing order of self-awareness, where the patient's request for care comes first; that is, this index characterizes the behavior of the patients most frequently, followed by the autonomy of the patent's behavior, the patient's awareness of the content of the request, the patient's awareness of the context in which she is acting, and lastly the patient's good relations with the others, which is the behavior least frequently presented by the subjects. The PPSA dimension was confirmed in relation to the two diagnoses (ADP and ADWP) and also longitudinally - that is, when the patients were evaluated in the pretreatment and posttreatment situations. As far as change is concerned, the self-awareness measure showed a significant improvement from the pre- to the posttreatment situation; that is, the patients' self-awareness was better as a whole after a period of treatment. This was true for both types of diagnoses, although considering the interaction pre- and posttreatment with diagnoses, biases were observed as far as autonomy and context were concerned. As far as autonomy was concerned, pretreatment, the ADWP patients appeared to be significantly less autonomous than the ADP patients. Most probably, the ADWP patients, being deeply involved in their affective problems, which in most cases were represented by depressive symptoms, were not able to express their subjectivity properly, whereas the ADP patients appeared to be aware of their request for help, showing that they were capable of elaborating their thoughts autonomously. The ADP patients' conditions upon entering the medical center might actually have been more critical than those of the ADWP patients. In the posttreatment situation, an opposite result was observed. The ADWP patients' self-awareness had significantly improved in comparison with the ADP patients', meaning that the clinical treatment resulted in better effects for patients who did not suffer from personality problems. Moreover, the results showed that in the posttreatment situation the ADP patients' selfawareness of the context was significantly better than that of the ADWP patients. MFRM probabilities were also calculated, confirming that there was a general improvement in the subjects' self-awareness behavior in the period of time considered. These results support the appropriateness of Rasch modeling with the data analyzed, an encouraging 
sign that the methodological approach taken in this study is appropriate when the need is to devise specific measurement tools, also, for example, for the analysis of behaviors in clinical psychology.

The results of this research, although they do not definitively define a self-awareness dimension, open new horizons for the study of the relationship between selfawareness and psychopathology, allowing the study of its connection with the effects of psychoanalytical/ pharmacological treatments on an empirical base.

\section{AUTHOR NOTE}

Correspondence concerning this article should be addressed to S. Mannarini, University of Padua, Department of General Psychology, Via Venezia 8, 35131 Padua, Italy (e-mail: stefania.mannarini@unipd.it).

\section{REFERENCES}

Birchwood, M., Smith, J., Drury, V., Healy, J., Macmillan, F., \& Slade, M. (1994). A self-report Insight Scale for psychosis: Reliability, validity and sensitivity to change. Acta Psychiatrica Scandinavica, 89, 62-67.

BLum, H. P. (1979). The curative and creative aspects of insight. Journal of the American Psychoanalytic Association, 27(Suppl.), 41-69.

Bond, T. G., \& Fox, C. M. (2001). Applying the Rasch model: Fundamental measurement in the human sciences. Mahwah, NJ: Erlbaum.

FreUd, A. (1979). The role of insight in psychotherapy: Introduction. In H. P. Blum (Ed.), Psychoanalytic explorations of technique: Discourse on the theory of therapy. New York: International Universities Press.

FreUd, S. (1975). Introduzione al narcisismo. In S. Freud, Opere: Vol. VII. 1912-1914 (pp. 443-472). Turin: Bollati Boringhieri. (Original work published 1914)

HATCHER, R. L. (1973). Insight and self-observation. Journal of the American Psychoanalytic Association, 21, 377-398.

Kennedy, H. (1979). The role of insight in child analysis: A developmental viewpoint. In H. P. Blum (Ed.), Psychoanalytic explorations of technique: Discourse on the theory of therapy. New York: International Universities Press.

KRIS, E. (1975). Selected papers. New Haven: Yale University Press.
LinaCre, J. M. (1989). Many-facet Rasch measurement. Chicago: MESA Press.

LinACRE, J. M. (2005). A user's guide to Facets Rasch model computer programs. Available at www.winsteps.com.

LoPEZ, D. (1983). La psicoanalisi della persona. Turin: Boringhieri.

Lopez, D., \& ZoRZI MenEguzzo, L. (1989). Dal carattere alla persona. In A. Semi (Ed.), Trattato di psicoanalisi. Milan: Raffaello Cortina Editore.

MANGINI, E. (2001). Insight come breccia di verità. IV ${ }^{\circ}$ Colloquio Psicoanalitico di Venezia.

Markova, I. S., \& Berrios, G. E. (1992). The meaning of insight in clinical psychiatry. British Journal of Psychiatry, 160, 850-860.

Markova, I. S., \& Berrios, G. E. (1995). Insight in clinical psychiatry: A new model. Journal of Nervous \& Mental Disease, 183, 743-751.

Neubauer, P. B. (1979). The role of insight in psychoanalysis. Journal of the American Psychoanalytic Association, 27(Suppl.), 29-40.

RANGELL, L. (1981). From insight to change. Journal of the American Psychoanalytic Association, 29, 119-141.

RASCH, G. (1980). Probabilistic models for some intelligence and attainment tests. Chicago: University of Chicago Press. (Original work published 1960)

Roncone, R., Tozzini, C., Mazza, M., De Risio, A., Giosú́, P., MoRosini, P., \& Cusacchia, M. (2003). Validation of the Italian version of the Self-Report Insight Scale. Epidemiologia e psichiatria sociale, 12, 63-75.

SACERDoti, G. (1986). Luci e ombre dell'insight teorico. Gli Argonauti, 8, 17-32.

Sacerdoti, G., \& Spacal, S. (1985). Insight. Rivista di Psicoanalisi, 31, 59-74.

SANDLER, J. (1979). Il paziente e l'analista. Rome: Astrolabio.

Silvestri, A., Lalli, R., Mannarini, S., Ferruzza, E., Nuzzaci, V., Furin, A. ET AL. (2008). Consapevolezza di sé: Definizione di una misura. Rivista di Psichiatria, 43, 376-386.

\section{NOTE}

1. The data were collected at the Casa di Cura Parco dei Tigli medical center, Teolo-Padua (Italy), in the years 2006 and 2007.

(Manuscript received January 20, 2009; revision accepted for publication April 10, 2009.) 